\title{
Metal-based Nanoparticles as antifungal and nematicidal agents
}

\author{
Panagiota Tryfon, Nathalie N. Kamou, Nikoletta Ntalli, Katerina Karamanoli, Urania \\ Menkissoglu-Spiroudi *, Catherine Dendrinou- Samara *.
}

\begin{abstract}
Nanotechnology provides unique opportunities for the development of novel nanotools in various fields, especially in agriculture. In terms of desired function, nanomaterials are proposed as environmentally acceptable substitutes that can lead to a green agricultural revolution. Among others, inorganic nanoparticles (INPs) stand at the first line. The advantage of INPs arises from their unique inherent physicochemical properties in combination with nanoeffects that classify them as nanofertilizers or nanopesticides. Thus, INPs have been found to enhance pesticide efficiency and provide crop productivity at low doses. Nanopesticides are considered less harmful for the environment because they are usually involved in the slow release of the active ingredient that is employed in less quantities of the formulate, compared to the conventional pesticides. Herein, INPS that are based on bioessential and bioactive elements, namely copper and zinc, have been prepared and tested as antifungal and nematicidal agents. Copper nanoparticles (Cu NPs), zinc oxide nanoparticles (ZnO NPs) and bimetallic nanostructures of CuZn and CuZn@ZnO NPs have been isolated through assisted microwave process and solvothermal method in the presence of polyols. Sizes were identified in a range of 20 to $40 \mathrm{~nm}$ while polyols serve as coatings to the INPs. Antifungal and nematicidal activity of NPs was studied in vitro against the phytopathogenic fungi Botrytis cinerea and Sclerotinia sclerotiorum as well as on the root-knot nematode Meloidogyne javanica. Lettuce plants infected either with S. sclerotiorum or M. javanica, were sprayed with the synthesized NPs. The photosynthetic quantum yield, net photosynthesis and chlorophyll content index of treated plants were also measured in order to investigate the photocatalytic activity of the NPs. The Cu-based metal NPs and bimetallic CuZn@ZnO NPs were identified as the most effective control agents against S. sclerotiorum and M. javanica and were constituted effective tools to prevent fungal and nematicide infections in agricultural field after further investigation.
\end{abstract}

Keywords: metal-based nanomaterials; antifungal agents; nematicides 